Editorial

\title{
Special Issue on New Horizons in Time Domain Diffuse Optical Spectroscopy and Imaging
}

\author{
Yoko Hoshi \\ Department of Biomedical Optics, Institute for Medical Photonics Research, Preeminent Medical Photonics \\ Education \& Research Center, Hamamatsu University School of Medicine, Hamamatsu, \\ Shizuoka 431-3192, Japan; yhoshi@hama-med.ac.jp; Tel.: +81-53-345-2329
}

Received: 17 March 2020; Accepted: 13 April 2020; Published: 16 April 2020

\section{Time Domain Measurements}

In 1977, Jöbsis first described the in vivo application of near-infrared spectroscopy (NIRS) [1], also called diffuse optical spectroscopy (DOS). Originally, NIRS was designed for clinical monitoring of tissue oxygenation, and today it has also become a useful tool for neuroimaging studies (functional near-infrared spectroscopy (fNIRS)) [2-4]. However, difficulties in the selective and quantitative measurements of tissue hemoglobin $(\mathrm{Hb})$, which have been central issues in the NIRS field for over 40 years, are yet to be solved. To overcome these problems, time domain (TD) $[5,6]$ and frequency domain (FD) $[7,8]$ measurements have been tried. Presently, a wide range of NIRS instruments are available, including commercial commonly available instruments for continuous wave (CW) measurements based on the modified Beer-Lambert law (steady-state domain measurements). Among these measurements, the TD measurement is the most promising approach, although, compared with $\mathrm{CW}$ and FD measurements, TD measurements are less common due to the need for large and expensive instruments with poor temporal resolution and limited dynamic range. However, thanks to technological developments, TD measurements are increasingly being used in research and also in various clinical settings $[9,10]$.

\section{Light Propagation in Biological Tissue and Time Domain Diffuse Optical Spectroscopy}

In TD DOS, also termed time-resolved spectroscopy (TRS), tissue is irradiated by ultrashort (picosecond order) laser pulses, and the intensity of the emerging light at the tissue surface is recorded over time to show a temporal point spread function (TPSF) with picosecond resolution. The mean total length of the light path is determined by multiplying the light speed in the media by the mean transit time of the scattered photons, which is calculated with the TPSF [5]. The TPSF reflects the propagation of light in biological tissue, which is characterized by the optical properties of absorption, scattering, scattering anisotropy, and refractive indexes. It is widely accepted that the radiative transfer equation (RTE) correctly describes the light propagation in biological tissue [11,12]. Since, however, the computational cost is extremely high in numerically solving the RTE, the photon diffusion equation (PDE), a diffusion approximation to the RTE, is often used. Based on the PDE, it is possible to estimate the absorption $\left(\mu_{a}\right)$ and reduced scattering $\left(\mu_{s}{ }^{\prime}\right)$ coefficients with the TPSF, and to calculate concentrations of biological chromophores, including $\mathrm{Hb}$. The TPSF also carries information about depth-dependent attenuation based on the correlation of the detection time with the penetration depth of photons. Accurate numerical modelling of light propagation is critical for the quantification of TD measurements and the image reconstruction of diffuse optical tomography (DOT), as will be described below. 


\section{Time Domain Diffuse Optical Tomography}

Diffuse optical tomography, one of the most sophisticated near-infrared optical imaging techniques for observations through biological tissue, allows 3-D quantitative imaging of optical properties which include functional and anatomical information [13]. The DOT image reconstruction can be approximately divided into two kinds-one is a linearization approach; the other is a non-linear iterative approach. With DOT, especially the non-linear iterative DOT, it is expected that it will become possible to overcome the limitations of conventional NIRS as well as it offers the potential for diagnostic optical imaging. The DOT algorithm essentially consists of two parts-one is a forward model to calculate the light propagation and the resultant outward re-emissions at the boundary of the tissue, typically based on the PDE or the RTE. The other is an inverse model to search for the distribution of optical properties. The implementation of DOT is possible with CW, TD, and FD measurements, where the TD measurements provide more of the information required for image reconstruction.

\section{Cutting Edge Time Domain Diffuse Optical Spectroscopy and Imaging}

This Special Issue highlights the issues at the cutting edge of TD DOS and DOT. It covers all aspects related to TD measurements described above, including advances in hardware, methodology, theory of light propagation, and clinical applications. The Special Issue has two reviews and 10 original research papers. One review paper by Yamada, Suzuki and Yamashita provides a comprehensive review of the past and current status of TD DOS and TD DOT, with chronological summaries of the major events in instrument and theoretical method developments [14]. This paper will help readers who are new to NIRS and also experts to obtain an overview of TD measurements and broaden their knowledge and understanding. The second review is by Lange and Tachtsidis and focuses on clinical applications in brain monitoring, where they also describe recent developments in instrumentation and methodologies that have the potential to affect and broaden the clinical use of TD measurements [15].

Five of 10 original papers deal with clinical applications that utilize the strengths of TD measurements. Ueda and Saeki applied TD DOS to three studies on breast cancer, reporting the detection rate of breast cancer, tumor hemodynamic responses to neoadjuvant chemotherapy, and antiangiogenic therapy [16]. Kinoshita et al., who measured skeletal muscle oxygenation in the lower extremities during $3 \mathrm{~h}$ of continuous sitting, report that compression stockings suppress increases in extracellular water in the lower extremities, leading to reduced blood volume and oxygenation levels in skeletal muscles [17]. The study by Morimoto et al. measured cerebral blood volume and optical properties in five term neonates from 2-3 min to $15 \mathrm{~min}$ after birth, and demonstrate that TD DOS can stably measure the cerebral hemodynamics of neonates in the labor room [18]. Sakatani et al. examined the effects of aging, cognitive dysfunction, and brain atrophy on $\mathrm{Hb}$ concentrations and optical pathlengths at rest in the prefrontal cortex in 202 elderly subjects, concluding that TD DOS enables an evaluation of the relation between prefrontal oxygenation at rest and cognitive function [19]. Kuroiwa et al. employed TD DOS to test their hypothesis that total $\mathrm{Hb}$ concentration in abdominal subcutaneous adipose tissue correlates negatively with risk factors for developing metabolic diseases and were able to verify the hypothesis [20].

Ohmae et al. conducted basic research into the application of TD measurements to breast cancer, and report that the validity of TD DOS measurements of the lipid and water contents of the breast is confirmed by a comparison of the TD DOS values to the values measured by dual-energy computed tomography [21]. The paper by Di Sieno et al. deals with hardware, a TD fast gated NIRS system, and presents results showing that the gating approach can improve the contrast and contrast-noise ratio for the detection of absorption changes, irrespective of the source-detector separation distance [22].

Three papers present theoretical studies. To recover the optical properties from boundary measurements, iterative inversion schemes, where a theoretical TPSF is derived from the analytical solution to the PDE and fitted to the measured temporal profile of detected light intensity, are often used [23]. However, in these schemes, the initial guesses need to be close to the true values. Jiang et al. propose a scheme combining Markov chain Monte Carlo and iterative methods to overcome this 
weakness in iterative schemes [24]. In TD DOT, regarding the datatypes obtained from the TPSF, such as temporal windows and Fourier transformations, determining which datatypes are used for image reconstruction is crucial for computational efficiency as well as for image quality. Orive-Miguel et al. propose a new process for the efficient computation of long sets of temporal windows in the FD and demonstrate that the absorption quantification of the inclusions in a rectangular medium is improved at all depths in numerical experiments by the proposed method [25]. The M-th order delta-Eddington equation (dEM) is used as one effective approach to reduce the computational cost of a numerical solution to the RTE. The final paper in the issue by Fujii et al. examined photon transport in 3D, homogeneous, highly forward-scattering media with different optical properties by using time-dependent RTE, dEM, and PDE and estimated the length and time scales in which the dEM is valid [26].

\section{Future Prospects and Challenges}

The ultimate goal of developing TD measurements is to establish an optical-based diagnosis. Further studies on the numerical modeling of light propagation in biological tissue, developing accurate and efficient inverse solutions and high-quality instruments are required for reaching this goal. Although these tasks are challenging, recent advances in computer and optical technologies will advance the efforts to solve these bottlenecks.

Funding: This research received no external funding.

Acknowledgments: I wish to express my very great appreciation to all of the authors and peer reviewers for their valuable contributions to this Special Issue. I also wish to thank the editorial team of Applied Sciences for their assistance. I am particularly grateful for the assistance given by Marin Ma, a section managing editor.

Conflicts of Interest: The authors declare no conflict of interest.

\section{References}

1. Jobsis, F. Noninvasive, infrared monitoring of cerebral and myocardial oxygen sufficiency and circulatory parameters. Science 1977, 198, 1264-1267. [CrossRef]

2. Hoshi, Y.; Tamura, M. Detection of dynamic changes in cerebral oxygenation coupled to neuronal function during mental work in man. Neurosci. Lett. 1993, 150, 5-8. [CrossRef]

3. Kato, T.; Kamei, A.; Takashima, S.; Ozaki, T. Human Visual Cortical Function during Photic Stimulation Monitoring by Means of near-Infrared Spectroscopy. Br. J. Pharmacol. 1993, 13, 516-520. [CrossRef]

4. Villringer, A.; Planck, J.; Hock, C.; Schleinkofer, L.; Dirnagl, U. Near infrared spectroscopy (NIRS): A new tool to study hemodynamic changes during activation of brain function in human adults. Neurosci. Lett. 1993, 154, 101-104. [CrossRef]

5. Delpy, D.T.; Cope, M.; Van Der Zee, P.; Arridge, S.R.; Wray, S.; Wyatt, J. Estimation of optical pathlength through tissue from direct time of flight measurement. Phys. Med. Boil. 1988, 33, 1433-1442. [CrossRef]

6. Chance, B.; Leigh, J.S.; Miyake, H.; Smith, D.S.; Nioka, S.; Greenfeld, R.; Finander, M.; Kaufmann, K.; Levy, W.; Young, M. Comparison of time-resolved and -unresolved measurements of deoxyhemoglobin in brain. Proc. Natl. Acad. Sci. USA 1988, 85, 4971-4975. [CrossRef]

7. Lakowicz, J.R.; Berndt, K. Frequency-domain measurements of photon migration in tissues. Chem. Phys. Lett. 1990, 166, 246-252. [CrossRef]

8. Duncan, A.; Whitlock, T.L.; Cope, M.; Delpy, D.T. A multiwavelength, wideband, intensity modulated optical spectrometer for near infrared spectroscopy and imaging. Proc. SPIE 1993, 1888, 248-257.

9. Wabnitz, H.; Taubert, D.R.; Mazurenka, M.; Steinkellner, O.; Jelzow, A.; Macdonald, R.; Milej, D.; Sawosz, P.; Kacprzak, M.; Liebert, A.; et al. Performance assessment of time-domain optical brain imagers, part 1: Basic instrumental performance protocol. J. Biomed. Opt. 2014, 19, 086010. [CrossRef] [PubMed]

10. Abdalmalak, A.; Milej, D.; Diop, M.; Shokouhi, M.; Naci, L.; Owen, A.M.; Lawrence, K.S. Can time-resolved NIRS provide the sensitivity to detect brain activity during motor imagery consistently? Biomed. Opt. Express 2017, 8, 2162-2172. [CrossRef] [PubMed]

11. Duderstadt, J.J.; Martin, W.R.; Aronson, R. Transport Theory. Phys. Today 1982, 35, 65. [CrossRef] 
12. Ishimaru, A. Wave Propagation and Scattering in Random Media; Institute of Electrical and Electronics Engineers (IEEE): New York, NY, USA, 1999.

13. Hoshi, Y.; Yamada, Y. Overview of diffuse optical tomography and its clinical applications. J. Biomed. Opt. 2016, 21, 91312. [CrossRef]

14. Yamada, Y.; Suzuki, H.; Yamashita, Y. Time-Domain Near-Infrared Spectroscopy and Imaging: A Review. Appl. Sci. 2019, 9, 1127. [CrossRef]

15. Lange, F.; Tachtsidis, I. Clinical Brain Monitoring with Time Domain NIRS: A Review and Future Perspectives. Appl. Sci. 2019, 9, 1612. [CrossRef]

16. Ueda, S.; Saeki, T. Early Therapeutic Prediction Based on Tumor Hemodynamic Response Imaging: Clinical Studies in Breast Cancer with Time-Resolved Diffuse Optical Spectroscopy. Appl. Sci. 2018, 9, 3. [CrossRef]

17. Kinoshita, M.; Kurosawa, Y.; Fuse, S.; Tanaka, R.; Tano, N.; Kobayashi, R.; Kime, R.; Hamaoka, T. Compression Stockings Suppressed Reduced Muscle Blood Volume and Oxygenation Levels Induced by Persistent Sitting. Appl. Sci. 2019, 9, 1800. [CrossRef]

18. Morimoto, A.; Nakamura, S.; Sugino, M.; Koyano, K.; Htun, Y.; Arioka, M.; Fuke, N.; Mizuo, A.; Yokota, T.; Kato, I.; et al. Measurement of the Absolute Value of Cerebral Blood Volume and Optical Properties in Term Neonates Immediately after Birth Using Near-Infrared Time-Resolved Spectroscopy: A Preliminary Observation Study. Appl. Sci. 2019, 9, 2172. [CrossRef]

19. Sakatani, K.; Hu, L.; Oyama, K.; Yamada, Y. Effects of Aging, Cognitive Dysfunction, Brain Atrophy on Hemoglobin Concentrations and Optical Pathlength at Rest in the Prefrontal Cortex: A Time-Resolved Spectroscopy Study. Appl. Sci. 2019, 9, 2209. [CrossRef]

20. Kuroiwa, M.; Fuse, S.; Amagasa, S.; Kime, R.; Endo, T.; Kurosawa, Y.; Hamaoka, T. Relationship of Total Hemoglobin in Subcutaneous Adipose Tissue with Whole-Body and Visceral Adiposity in Humans. Appl. Sci. 2019, 9, 2442. [CrossRef]

21. Ohmae, E.; Yoshizawa, N.; Yoshimoto, K.; Hayashi, M.; Wada, H.; Mimura, T.; Asano, Y.; Ogura, H.; Yamashita, Y.; Sakahara, H.; et al. Comparison of Lipid and Water Contents by Time-domain Diffuse Optical Spectroscopy and Dual-energy Computed Tomography in Breast Cancer Patients. Appl. Sci. 2019, 9, 1482. [CrossRef]

22. Di Sieno, L.; Mora, A.D.; Torricelli, A.; Spinelli, L.; Re, R.; Pifferi, A.; Contini, D. A Versatile Setup for Time-Resolved Functional Near Infrared Spectroscopy Based on Fast-Gated Single-Photon Avalanche Diode and on Four-Wave Mixing Laser. Appl. Sci. 2019, 9, 2366. [CrossRef]

23. Patterson, M.S.; Chance, B.; Wilson, B.C. Time resolved reflectance and transmittance for the non-invasive measurement of tissue optical properties. Appl. Opt. 1989, 28, 2331-2336. [CrossRef]

24. Jiang, Y.; Hoshi, Y.; Machida, M.; Nakamura, G. A hybrid inversion scheme combining Marcov chain Monte Carlo and iterative methods for determining optical properties of random media. Appl. Sci. 2019, 9, 3500. [CrossRef]

25. Orive-Miguel, D.; Herve, L.; Condat, L.; Mars, J. Improving Localization of Deep Inclusions in Time-Resolved Diffuse Optical Tomography. Appl. Sci. 2019, 9, 5468. [CrossRef]

26. Fujii, H.; Ueno, M.; Kobayashi, K.; Watanabe, M. Characteristic Length and Time Scales of the Highly Forward Scattering of Photons in Random Media. Appl. Sci. 2019, 10, 93. [CrossRef]

(C) 2020 by the author. Licensee MDPI, Basel, Switzerland. This article is an open access article distributed under the terms and conditions of the Creative Commons Attribution (CC BY) license (http://creativecommons.org/licenses/by/4.0/). 